\title{
Coulomb fission in multiply-charged ammonia clusters: Accurate measurements of the Rayleigh instability limit from fragmentation
} patterns.

Christopher Harris and Anthony J. Stace

Department of Physical and Theoretical Chemistry, School of Chemistry, University of Nottingham, University Park, Nottingham NG7 2RD, U.K.

\begin{abstract}
A series of experiments have been undertaken on the fragmentation of multiply charged ammonia clusters, $\left(\mathrm{NH}_{3}\right)_{n}{ }^{z+}$, where $z \leq 8$ and $n \leq 800$, to establish Rayleigh instability limits, whereby clusters at certain critical sizes become unstable due to Coulomb repulsion between the resident charges. The experimental results are in excellent agreement with theoretical predictions of critical size. Electrostatic theory has been used to help identify fragmentation patterns on the assumption that the clusters separate into two dielectric spheres. The predicted Coulomb repulsion energy is then used to match individual peak profiles recorded in the experiments in order to establish fragment size and the amount of charge they carry. The results show that fragmentation is very asymmetric in terms of both the numbers of molecules involved and the amount of charge each fragment accommodates. It is suggested that the results support the ion evaporation model that is frequently used to interpret electrospray experiments.
\end{abstract}

\section{Introduction}

The first quantitative interpretation concerned with the stability of multiply charged clusters and droplets was presented in 1882 by Lord Rayleigh. ${ }^{1}$ Rayleigh proposed that, as a consequence of electrostatic repulsion, a charged spherical mass of liquid could move from 
being in a state of stable equilibrium to becoming unstable as the magnitude of the charge is increased. In many respects, multiply charged clusters of molecules held together by van der Waals forcers are ideal models with which to test this theory. They can be both size- and charge-selected and the retaining forces are often sufficiently weak as to make clusters susceptible to subtle changes in the strength of the Coulomb interaction. Applications to clusters carrying $z$ units of charge are most frequently described in terms of a minimum number of molecules, $n_{\text {crit }}(z)$, required to suppress fission due to Coulomb repulsion: ${ }^{2-7}$

$$
n_{\text {crit }}(z)=\frac{(z e)^{2}}{64 \pi^{2} \gamma \varepsilon_{0} r_{m}^{3}}
$$

Where $e$ is the elementary unit of charge, $\varepsilon_{0}$ is the permittivity of free space, $\gamma$ is the surface tension of the molecular liquid and $r_{m}$ is the radius of a constituent atom or molecule. Below $n_{\text {crit }}(z)$ a cluster is considered to be unstable with respect to fission via charge separation. Earlier studies of Coulomb fission made use of collisional activation to reduce the size of a stable dication or trications to a point where Coulomb fission could be observed to promote fragmentation via charge separation. ${ }^{8-10}$ More recently, Coulomb fission has been found to occur through the metastable or unimolecular decay of mass-selected dication and trications molecular clusters. ${ }^{11-13}$ These observations have shown that the decay process can be both delayed $\left(\sim 10^{-4} \mathrm{~s}\right)$ and very asymmetric; a typical example being $\left(\mathrm{NH}_{3}\right)_{52} \mathrm{H}_{2}{ }^{2+} \rightarrow\left(\mathrm{NH}_{3}\right)_{40} \mathrm{H}^{+}+$ $\left(\mathrm{NH}_{3}\right)_{12} \mathrm{H}^{+},{ }^{11-13}$ and it has been possible to interpret these experiments in terms of a two-body Coulomb fission step, for which a generalised schematic description is given in Figure 1. Here, a spherical cluster carrying a charge of $z^{+}$fragments into two smaller spherical units carrying charges of $y^{+}$and $x^{+}$.

In the examples discussed both here and in earlier experiments, ${ }^{11-13}$ the intrinsic energy barrier to fission, $E_{\text {barrier }}$, is overcome by the presence of residual internal energy remaining in the precursor cluster following ionisation. It is assumed that this energy is randomly distributed among the many vibrational modes within the cluster, but that at some point in time, a fraction 
of that energy will reside in a collective mode that will drive the cluster over the fission barrier. Two important pieces of information are recorded during these experiments, and these are the fragmentation pattern and the centre-of-mass kinetic energy released as the two fragments shown in Figure 1 separate. For all of the experiments undertaken thus far, a very close match has been found between the experimental kinetic energy release measurements, $K E R$, and the calculated maximum electrostatic potential energy between the two charged spherical fragments as they separate (see Figure 1). ${ }^{11-13}$ Preliminary electrostatic calculations on ammonia dications were undertaken using a model due to Linse, ${ }^{14}$ but more recently new theory has been formulated to treat electrostatic interactions between charged dielectric materials. ${ }^{15,16}$ For the case of quadruply charged clusters, the theory has also been used to differentiate between symmetric and asymmetric charge separation. ${ }^{13}$ These comparisons between experiment and theory are only concerned with events that take place once the two fragments start to separate; no information is provided as to the magnitude of $E_{\text {barrier. However, the very }}$ good agreement with theory does suggest that during the time the fragments experience mutual Coulomb repulsion, there is very little energy partitioning to internal (intermolecular) degrees of freedom

The principal focus of the work reported here has been to provide an accurate measure of the Rayleigh instability limit by monitoring the Coulomb fission of multiply charged ammonia clusters with $z$ in the range 2-8. The numbers recorded are then compared with values for $n_{\text {crit }}(z)$ calculated from equation (1). However, as part of this process, information has also been recorded on the fragmentation patterns of multiply charged clusters, where as part of the analysis, detailed electrostatic calculations have been undertaken to help interpret the data regarding the degree of asymmetry exhibited by the fragments both in terms of size and charge. In some respects, the patterns of behaviour exhibited by multiply charged clusters should have features in common with decay channels that have proposed for highly charged ions during 
electrospray ionization (ESI), ${ }^{17-29}$ and in this context discussion has focused two mechanisms. For highly charged proteins a charge residue model CRM) has been proposed ${ }^{26,27}$ whereby extensive (neutral) solvent evaporation leaves the ion holding a significant fraction of the available charge. In contrast, the ion evaporation model (IEM) is thought to proceed via the ejection of small solvent ions, and may be more appropriate under circumstances where residue ions are found to carry just a fraction of the initial charge generated during ESI. ${ }^{28,29}$

\section{EXPERIMENTAL SECTION}

Observations on the fragmentation patterns of multiply charged molecular clusters have been made on an apparatus that combines a high resolution reversed geometry mass spectrometer (VG Analytical ZAB-E) with a pulsed supersonic cluster source. Since details of the experimental procedure have been given previously, ${ }^{12}$ what follows is a brief summery. Neutral molecular clusters of the form $\left(\mathrm{NH}_{3}\right)_{n}$ were generated via the adiabatic expansion of a gaseous mixture of ammonia and argon through a pulsed, conical nozzle, with the ammonia content being between $1 \%$ and 5\%. The resultant neutral clusters were ionized by $70-100 \mathrm{eV}$ electrons and the ion beam extracted from the ion source at a potential of either $+6 \mathrm{kV}$ or +7 $\mathrm{kV}$ into the flight tube of the mass spectrometer. Cluster ions with a particular combination of charge $\left(z_{1}\right)$ and mass $\left(m_{1}\right)$ were selected using a magnet and the ionic products of Coulomb fission in the field free region between the magnet and an electrostatic analyser (ESA) were identified by scanning the voltage on the latter. The field-free region is $1.5 \mathrm{~m}$ in length and ions are approx. $5 \times 10^{-5} \mathrm{~s}$ old when they enter that section of the mass spectrometer. This linkscanning procedure provides a mass-analysed ion kinetic energy (MIKE) spectrum, ${ }^{30}$ which can be used to identify ionic fragments according to their laboratory-frame kinetic energy, and the energy spread of a peak can be related to the centre-of-mass kinetic energy released during 
fragmentation. ${ }^{30}$ To detect the principal charged product from the fission of a multiply charged cluster, the ESA has been scanned to record ionic fragments with laboratory-frame kinetic energies from $10 \mathrm{keV}$ downwards. For laboratory-frame kinetic energies of between $7 \mathrm{keV}$ and $10 \mathrm{keV}$ there are no background ion signals from other processes, such as the loss of neutral molecules, which means the very weak signals that arise from Coulomb fission can be recorded without interference. However, this approach does mean that for each decay step, only the larger of the two charged fragments is detected. The size of the smaller fragment is determined from mass and charge balance, together with the assumption that it emerges as a single unit, which is supported by the shapes of peak profiles recorded following Coulomb fission in doubly and triply charged clusters. ${ }^{12,13}$ Attempts to record data on the smaller of the two fragments were hampered by two factors: (i) the severe instrumental discrimination light ions with high kinetic energies can experience; and (ii) an overlap with peaks arising from the loss of neutral molecules, which has previously been shown to compete with Coulomb fission. ${ }^{12}$

From the magnitude of the electric sector voltage necessary to transmit them, the mass-tocharge ratio of fragment ions can be identified from the following equation: ${ }^{30}$

$$
E^{*}=\frac{m_{2}}{m_{1}} \frac{z_{1}}{z_{2}} E_{0}
$$

$E_{0}$ is the initial parent ion kinetic energy $(6$ or $7 \mathrm{keV}), E^{*}$ is the kinetic energy recorded following fragmentation and $m_{2}$ and $z_{2}$ are the mass and charge, respectively, of the fragment ion being detected. Ions were detected with a Daly scintillation detector linked to a lock-in amplified (Stanford Research Systems SR850), which provided phase-sensitive detection referenced with respect to a train of nozzle pulses. During the course of these experiments, the background pressure beyond the ion source remained below $1 \times 10^{-7} \mathrm{mbar}$, thus ensuring minimal interference from collision induced fragmentation. 
An added complication in the study of Coulomb fission in multiply-charged molecular clusters, such as those of water or ammonia, where there are labile protons, is that during ionisation and/or fragmentation in the ion source, protons are either readily lost or acquired by potential precursor ions. ${ }^{31,32}$ As a result, mass spectra show that cluster ions can take on multiple forms, often separated by the mass-to-charge ratio of a single proton. ${ }^{31,32}$ In the case of ammonia, Garvey and co-worker have shown there are two dominate doubly charged ions and three prominent triply charged ions together with a less intense non-protonated form. ${ }^{33,34}$ The authors propose that, following ionization, protonation in both examples comes from a sequence of internal ion-molecule reactions. ${ }^{33,34}$ However, as a consequence of proton mobility, an increase in charge $(z)$ on a cluster, reduces the ability to resolve and identify individual ions separated by just $\mathrm{H} / z$, particularly, when it is necessary to retain sufficient ion signal to record a fragmentation pattern. A further complication arises during Coulomb fission, because this process may also involve fragments losing or gaining a proton(s), which then compromises the identification of their exact composition.

The approximate timescale over which the mass spectrometer allows observations to be made on fragmentation, $\sim 50-100 \mu \mathrm{s}$, places limitations on the types of processes that might be recorded and the accuracy with which various size limits, such as those associated with Rayleigh instability, can be determined. Very small clusters that experience high levels of ionisation can be expected to undergo spontaneous Coulomb fission before they have time to leave the ion source, $\sim 1 \mu \mathrm{s}$. The next point of travel is the region between the ion source and the magnet; again events occurring here cannot be monitored using the linked scanning procedure adopted here, but would correspond to cluster ions that are sufficiently stable to leave the ion source, but still have comparatively short lifetimes. Thus, any value recorded for a minimum critical size, $n_{c r i t}(z)$, from fragmentation in the region between the magnet and the electric sector will be an upper limit, because clusters of size, for example, $n_{\text {crit }}(z)-1$ 
will have fragmented before reaching that observation point. Likewise, there will be a certain size, $n_{s t a b}(z)$, at which fission ceases to be observed, but this will be a lower limit because if observations could be made, for example, on $n_{\text {stab }}(z)+1$, on a much longer timescale, then this ion might also be found to exhibit Coulomb fission.

In equation 1, the radii of ammonia clusters were calculated using the density of the bulk material in liquid phase $\left(0.73 \mathrm{~kg} \mathrm{~m}^{-3}\right)$ and a room temperature value of $0.038 \mathrm{Nm}^{-1}$ has been used for the surface tension.

\section{Results and discussion}

Figure 2 shows an example of a MIKE scan recorded from the doubly charged cluster ion $\left(\mathrm{NH}_{3}\right)_{52}{ }^{2+}$ where the loss of individual fragments is resolved and can be identified. The signal to noise ratio is typical of most of the experiments undertaken in this study. Also shown are the results of peak profile simulations that have been undertaken using a computer model of the flight path and geometry of those regions of the mass spectrometer in which ion fragmentation and detection take place. ${ }^{13}$ This procedure has been adopted in order to extract accurate kinetic energy releases from the experimental data for the purposes of comparison with electrostatic theory. Table 1 lists average kinetic energy releases, $\langle K E R\rangle$, as determined from a visual match between the experimental peak profiles in Figure 2 and the results of computer simulations. These numbers are considered to be more accurate than those quoted previously, ${ }^{11,12}$ which were determined from the FWHM (Full Width Half Maximum) of each peak. ${ }^{30}$ Also shown in Table 1 are potential energy maxima, $U_{\max }$, calculated from electrostatic theory for each of the fragmentation channels identified in Figure 2. Input to the theory consists of the radii of the fragments, their charge and the dielectric constant of the bulk material. Whilst it is acknowledged that the latter property may not be applicable to finite sized collections of 
molecules, in the absence of a better understanding of the multipole properties of clusters, the dielectric constant remains a useful parameter.

As can be seen from Table 1, the maximum repulsion calculated from the Coulomb potential energy curve, $U_{\max }$, is an excellent match to the experimental kinetic energy release data recorded for doubly charged ammonia clusters. In the analysis that follows, it will become evident that a similar quantitative interpretation of experimental data from the fission of more highly charged clusters is not possible. Therefore, based on the results in Table 1 and previous successful use of the electrostatic model, ${ }^{11-13}$ it is proposed that the process be inverted and that a value calculated for $U_{\max }$ for a given charge state and fission pattern is an accurate measure of the expected kinetic energy release. As such, values of $U_{\max }$ in conjunction with the simulation process will be used in the analysis of peak profiles recorded for fission processes associated with $z$ in the range $3-8$.

Previous measurements on the presence of triply charged ammonia clusters in mass spectra have identified the smallest observed size as being $\left(\mathrm{NH}_{3}\right)_{121}{ }^{3+} \cdot{ }^{34}$ However, by successively tuning the magnet to $\mathrm{m} / \mathrm{z}$ values that equate to triply charged clusters below this value and performing MIKE searches for characteristic fission signals, it is possible to identify $\left(\mathrm{NH}_{3}\right)_{116^{3+}}$ as being the smallest observed size (Table 2 gives a full list of critical sizes recorded for each of the multiply charged clusters studied). The possibility that these signals arise from close lying or coincident doubly charged cluster peaks can be ruled out as their equivalent $\mathrm{m} / \mathrm{z}$ values would correspond to approximately $\left(\mathrm{NH}_{3}\right)_{77^{2+}}$, which is well above the minimum stable size $\left(n_{\text {stab }}(2)=54\right.$; see Table 3$)$. As mentioned previously, a reduction in resolving power is required to achieve the necessary ion signal for the detection of fragments. Consequently, the magnetic sector is not able to select individual ion species separated by $1 / 3 \mathrm{amu}(\mathrm{H} / z)$; therefore, it is assumed that the resultant fission signals will have contributions from a number of protonated forms of the precursor cluster, together with any new combinations that may arise 
as a result of Coulomb fission / chemical reactivity. Figure 3 shows a MIKE scan recorded from a cluster ion in the region of $n=122$, where a high resolution mass spectrum shows the most intense precursor ion to be $\left(\mathrm{NH}_{3}\right)_{122-1}\left(\mathrm{NH}_{4}\right)^{3+}$ (a similar observation can be seen in mass spectra presented by Peifer et $a l .^{34}$ ). If Coulomb fission were to produce just two protonated forms of doubly charged fragment, for example, $\left(\mathrm{NH}_{3}\right)_{122-k-1}\left(\mathrm{NH}_{4}\right)^{2+}$ and $\left(\mathrm{NH}_{3}\right)_{122-k-2}\left(\mathrm{NH}_{4}\right)_{2}{ }^{2+}$, then, in the absence of any significant kinetic energy release, these fragments would be separated in a MIKE spectrum by a laboratory-frame-kinetic energy of less than $10 \mathrm{eV}$. Since the energy resolving capability of the ESA at low resolution is approximately $20 \mathrm{eV}$, it is clear that these individual fragments will not be resolved. The MIKE spectrum in Figure 3 shows the presence of four partially resolved features; a result which contrasts with previous measurements on the triply-charged clusters: $\left(\mathrm{C}_{6} \mathrm{H}_{6}\right)_{n}{ }^{3+},\left(\mathrm{CH}_{3} \mathrm{CN}\right)_{n}{ }^{3+}$ and $\left(\mathrm{C}_{4} \mathrm{H}_{8} \mathrm{O}\right)_{n}{ }^{3+}$, where sequences of fragments from Coulomb fission were clearly resolved. ${ }^{13}$ To further illustrate the point made above regarding proton transfer, the scan in Figure 3 is overlain with the predicted positions of fragments from possible reaction pathways that are separated by just $\mathrm{H} / z$. Based on the observations of Peifer et al., ${ }^{34}$ the doubly charged ion that is detected could take one of three possible forms. It can either be un-protonated, singly, or doubly protonated, and the lines on the spectrum indicate the laboratory-frame energies at which these various fragments would be detected. None of the steps shown has any kinetic energy release associated with it; a factor that will clearly complicate any additional analysis. Electrostatic theory gives a value for $U_{\max }$ as $1.62 \mathrm{eV}$; however, attempts to fit any of the features in Figure 3 with an equivalent simulated profile have not been successful. A qualitative conclusion derived purely from the overall position of peaks in the MIKE scan, is that $\left(\mathrm{NH}_{3}\right)_{122}{ }^{3+}$ and related ions within $\pm \mathrm{H}$ undergo a very asymmetric mode of Coulomb fission involving the loss of units containing between 11 and 13 , or possibly 14 , molecules. 
Given the difficulties outlined above concerning the precise identification of multiply charged ammonia clusters it is clear that for clusters where $z$ is $\geq 4$, a combination of the narrow mass spread between peaks in a mass spectrum and the expected reduction in energy resolution with increasing fragment mass, make it is impossible to resolve individual precursor ions and their associated fragments. Therefore, for ammonia clusters where $z$ is $\geq 4$, the identification of a critical size, $n_{\text {crit }}$, has been achieved by an alternative approach, whereby the reduction and eventual loss of any characteristic Coulomb fission signal originating from (unresolved) charged fragments has been monitored as a function of decreasing cluster size. To illustrate how this procedure works, Figure 4 shows a strong, but unresolved signal recorded following a MIKE scan of the fission products from the (assumed) precursor ion $\left(\mathrm{NH}_{3}\right)_{210}{ }^{4+}$. This is measurement was undertaken by "parking” the magnet on the mass-coincident ion $\left(\mathrm{NH}_{3}\right) 105^{2+}$, which is easily identified in a mass spectrum. A reduction in size by two molecules can be realised by "parking" on $\left(\mathrm{NH}_{3}\right)_{52}{ }^{+}$, which is coincident with $\left(\mathrm{NH}_{3}\right)_{208}{ }^{4+}$. Further mass selection can achieved by identifying similar $m / z$ ratios that coincide with +4 ions in the critical Rayleigh instability region. Since the mass range over which ions are known to exhibit Coulomb fission is narrow, there should be no interference in a MIKE scan from other mass-coincident multiply charged ions, for example, $\left(\mathrm{NH}_{3}\right)_{104}{ }^{2+}$ and $\left(\mathrm{NH}_{3}\right)_{156}{ }^{3+}$. The results have been used to construct a composite MIKE spectrum of the type shown in Figure 5, from where a critical size can be identified as $\left(\mathrm{NH}_{3}\right)_{205}{ }^{4+}$, and below this limit +4 clusters are too unstable to be observed. The result for $n=205$ was obtained by interpolation between $\left(\mathrm{NH}_{3}\right)_{51}{ }^{+}$and $\left(\mathrm{NH}_{3}\right)_{103}{ }^{2+}$.

As already noted, in these experiments it is the larger of the two fragments that is detected; therefore, in the case of a +4 cluster, the major fragment can carry a charge of either +3 or +2 , depending on how charge separation proceeds. Previous experiments on $\left(\mathrm{C}_{6} \mathrm{H}_{6}\right)_{n}{ }^{4+}$, $\left(\mathrm{CH}_{3} \mathrm{CN}\right)_{n}{ }^{4+}$ and $\left(\mathrm{C}_{4} \mathrm{H}_{8} \mathrm{O}\right)_{n}{ }^{4+}$ showed that these quadruply charged ions all favoured the formation of +3 fragments. From Eq. 2 it can be shown that for the route 


$$
\left(\mathrm{NH}_{3}\right)_{210}^{4+} \rightarrow\left(\mathrm{NH}_{3}\right)_{210-k}^{3+}+\left(\mathrm{NH}_{3}\right)_{k}^{+}
$$

the upper and lower limits to the signal in Fig. 4 correspond to $k$ lying in the range $12-20$. From electrostatic theory the Coulomb barrier, $U_{\max }$, generated from the interaction represent by Eq. 3 would be $\sim 2.2 \mathrm{eV}$. In contrast, if it is assumed that each fragment carries a charge of +2 , then $k$ would lie in the range $79-82$ and the corresponding electrostatic barrier would be $\sim 2.5 \mathrm{eV}$. The consequences of fragments having these two distinct pathways are very obvious from a simulation of the respective fragment ion peak profiles. Figure 4 shows a sequence of profiles calculated for the proposed fragments from Eq. (3) using an average kinetic energy release equal to $U_{\max }$. In contrast, if it is assumed that the two fragments are both large doubly charged clusters and are accompanied by a corresponding increase in energy release, then the resultant simulated profiles shown in Fig. 6 are distinctly dish-shaped, for which there is no evidence in the experimental data. The marked difference between the two results (Figure 4 compared with Figure 6) arises from a combination of a higher kinetic energy release and the detection of a lighter fragment. In the case of a triple charged fragment it is $\sim\left(\mathrm{NH}_{3}\right)_{195}{ }^{3+}$ being detected, whereas for the +2 route the detected fragment would be $\sim\left(\mathrm{NH}_{3}\right)_{130}{ }^{2+}$ and the latter would be subject to greater instrumental discrimination. An upper limit to Coulomb instability occurring in the timescale of the experiment can be determined by performing a similar experiment to that described above, but this time recording the size of cluster required to stabilise against Coulomb fission. Figure 7 shows an overlay of MIKE spectra which shows that beyond the precursor $\left(\mathrm{NH}_{3}\right)_{226}{ }^{4+}$ the signal strength becomes gradually weaker, with a stable size being assigned as $\left(\mathrm{NH}_{3}\right)_{234}{ }^{4+}$, which shows no evidence of any fission products. For the purposes of comparison, the fission profile for $\left(\mathrm{NH}_{3}\right)_{210}{ }^{4+}$ has been included in the overlay. 
An analysis of data recorded for $\left(\mathrm{NH}_{3}\right)_{n}{ }^{5+}$ using the approach outlined above gives a value for $n_{\text {crit }}(5)$ of 302 , but with a size range over which Coulomb instability is observed that is much larger than is seen in earlier examples (see Table 3). Figure 8 shows the result of a MIKE scan for $\left(\mathrm{NH}_{3}\right)_{385}{ }^{5+}$. The laboratory-frame kinetic energy spread spanned by the fragments is similar to that recorded for the +4 ion shown in Figure 4; however, what is immediately evident from an application of Eq. 2, is that no part of the peak can be attributed to the loss of singly charged ions. Figure 8 also shows how the position and width of the profile can accommodate a number of fragmentation channels involving the loss of doubly charged ions. Electrostatic theory predicts that these channels should be associated with a kinetic energy release of $\sim 3 \mathrm{eV}$ and individual profiles calculated for nine such fragmentation pathways are presented. Although the profiles are all predicted to be dish-shaped (a combination of light fragment mass and high energy release), there is both sufficient overlap and uncertainty in the masses of the fragments (addition and/or loss of multiple protons) that the absence of any similar features in the composite peak is not too unexpected.

Because of a shift in laboratory-frame kinetic energy seen in the fragmentation pattern for +5 ions, fragments arising from $\left(\mathrm{NH}_{3}\right)_{n}{ }^{6+}$ ions exhibit an overlap within the available ESA scan range such that both +5 and +6 fragments can be recorded simultaneously. Figure 9 shows an example that illustrates the decline in intensity of +5 fragment ions and the onset of stability at $\left(\mathrm{NH}_{3}\right)_{410}{ }^{5+}$, and the increase in intensity of Coulomb fission fragments from +6 ions starting at $\left(\mathrm{NH}_{3}\right)_{468}{ }^{6+}$. Fragments from the latter that are capable of detection could carry a charge of either $+3,+4$, or +5 ; however, similar to what has been found for the $\left(\mathrm{NH}_{3}\right)_{n}{ }^{5+}$ ions, no part of any of the recorded profiles could be assigned to +5 fragments. Table 4 lists the remaining options together with their calculated Coulomb energy barriers. Figure 10 shows profile calculated for fragments from $\left(\mathrm{NH}_{3}\right)_{492}{ }^{6+}$ where it has been assumed that the decay pattern follows that determined for $\left(\mathrm{NH}_{3}\right)_{n}{ }^{5+}$ ions and involves the loss of +2 ions to give a series of 
+4 ions that are detected. Each profile has been calculated with an average kinetic energy release of $\sim 4.0 \mathrm{eV}$. If instead, it is assumed that fragmentation follows the second of the pathways given in Table 4, then a profile calculated for a single fragment, $\left(\mathrm{NH}_{3}\right)_{300}{ }^{3+}$, with a predicted kinetic energy release of $\sim 4.2 \mathrm{eV}$ provides a very good match to the entire peak; however, given the patterns observed in previous examples, a single fragmentation step would seem unlikely.

A composite plot similar to those given in Figures 7 and 9 has been recorded for overlapping fragmentation patterns from +6 and +7 ions. The plot reveals $n_{\text {crit }}(7)$ to be 630 and the size at which +6 ions become stable as 564 . Potential fragmentation patterns for the +7 ion are the same as those discussed for the +6 ions and could involve the loss of $+1,+2$, and +3 ions. Figure 11 shows a fragment ion profile recorded for $\left(\mathrm{NH}_{3}\right)_{679}{ }^{7+}$, and it can be shown from Eq. 1 that, in contrast to the last two example, at least part of the peak could also be accounted for by the loss of singly charged fragments in the range $\left(\mathrm{NH}_{3}\right)_{2}{ }^{+}-\left(\mathrm{NH}_{3}\right)_{14}{ }^{+}$. However, there is the possibility of a more significant contribution from the loss of +2 ions as fragments and a series of simulated profiles for these channels are given in Figure 11, where each profile has associated with it an average kinetic energy release of $4.6 \mathrm{eV}$.

For the third possible charge separation pathway, one which would lead to the detection of +4 fragments, possible routes are:

$$
\begin{aligned}
& \left(\mathrm{NH}_{3}\right)_{665^{7+}} \rightarrow\left(\mathrm{NH}_{3}\right)_{442}{ }^{4+}+\left(\mathrm{NH}_{3}\right)_{223}{ }^{3+} \\
& \left(\mathrm{NH}_{3}\right)_{664}{ }^{7+} \rightarrow\left(\mathrm{NH}_{3}\right)_{438}{ }^{4+}+\left(\mathrm{NH}_{3}\right)_{226}{ }^{3+}
\end{aligned}
$$

Theory predicts the Coulomb barrier for these processes to be $\sim 5.2 \mathrm{eV}$; however, as before, the simulated peak profiles have very pronounced dish-shapes, which should be apparent in the experimental result if these fragmentation steps were taking place. 
MIKE spectra following the Coulomb fission from ammonia clusters with a charge of +8 have been recorded; however, the identification and analysis of these data is hampered by very poor signal strength making the composition of peaks somewhat difficult to interpret. If total ionisation proceeds through a sequence of single molecule events, then the total energy needed to remove eight electrons would be $74.3 \mathrm{eV}$, which is approaching the $100 \mathrm{eV}$ upper electron kinetic energy limit when the ion source is operated via "trap control". However, exothermic $\mathrm{NH}_{3}{ }^{+}$proton transfer reactions of the type discussed by Peifer et al. ${ }^{34}$, could provide additional internal energy to drive fragmentation. For a cluster containing 8 charges there are 4 possible modes of fission: $+7 /+1,+6 /+2,+5 /+3$ and $+4 /+4$. Calculated values of $U_{\max }$ for each of these channel in conjunction with the precursor ion $\left(\mathrm{NH}_{3}\right)_{824}{ }^{8+}$ are given in Table 5. Simulations show that it is possible to discount the fission channels leading to $+5 /+3$ and $+4 /+4$ products, as the simulated peaks are too wide, which leaves $+7 /+1$ and $+6 /+2$ as possibilities. A single peak simulated for $+7 /+1$ is narrower than the profile seen in the MIKE spectrum, which suggests a limited range of fragments, centred on $\left(\mathrm{NH}_{3}\right)_{31}{ }^{+}$, could be responsible for the observed peak. In contrast a single profile calculated for the $+6 /+2$ channel from the data given in Table 5 takes up almost the entire width of the experimental feature. Given what has been observed for the other multiple-charged ions, it would seem unlikely that there should be a single decay channel for the +8 ion; therefore it seems more likely that the experimental feature is composed predominantly from +7 fragments.

From the fragmentation data recorded above it has been possible to identify boundaries within which Coulomb fission is observed for a series of mass-selected $\left(\mathrm{NH}_{3}\right)_{n}{ }^{z+}$ ions. Although the events being observed are constrained by a specific time window, it is still possible to make comparisons with calculated Rayleigh instability limits on the understanding that the experimental values represent upper limits. Experimental and calculated values of $n_{\text {crit }}(z)$ are given in Table 2 and the results are plotted in Figure 12 together with data taken from Ref. 1. 
As can be seen, there is remarkably good agreement right across the data set between the experimental results and values calculated from the Rayleigh instability limit. In one respect, this level of agreement is unexpected in that the Rayleigh expression takes no account of the dielectric constant of the material concerned. In the case of ammonia the latter value is 22 and would represent a significant degree of shielding between the charges, which in turn should serve to reduce the value of $n_{\text {crit }}(z)$. However, such a reduction could be counteracted by the decline in surface tension predicted for finite-sized collections of atoms and molecules. ${ }^{35-37}$ Previous experiments on doubly and triply charged neon clusters also provide evidence of a close match between values recorded for $n_{\text {crit }}(z)$ and those calculated from Eq. (1). ${ }^{6}$

Finally, Fig. 13 examines the link between those charge separation pathways that are available for clusters carrying a charge of up to +8 , and those pathways that appear to account for the observations reported here. The data points are presented as a function of $z_{1} z_{2}$, which is taken to represent the nominal charges on the fragment ions, and also reflects the pure Coulomb term in the interaction potential between fragments as they separate. Plotted against the values are the calculated heights of the Coulomb barrier responsible for kinetic energy release $\left(U_{\max }\right)$. Shown below each experimental result is the smaller of the two charges to appear on the fission products. Since the +4 precursor ion represents the first opportunity for a choice of pathways, that result is probably the most suitable reference point from which to view the remaining results. That being the case, then the fragmentation patterns appear to strongly favour chargeasymmetric pathways, which in turn are associated with comparatively low Coulomb barriers. The data would also appear to support a drift towards +2 fragments as the charge on the precursor ion increases. However, the observations represented by Fig. 13 will be influenced by two factors. First, the experiments record delayed fragmentation pathways that occur on a $\sim 10^{-4} \mathrm{~s}$ timescale and that more energetic processes at short times may involve pathways with more symmetric charge separation that involve higher Coulomb barriers. Secondly, any 
pathway where both fragments are multiply charged has to yield ions that contain more molecules than $n_{\text {crit }}(z)$, which is certainly the case for those processes observed in these experiments.

Although there is some uncertainty regarding the exact nature of the fragmentation patterns, the data would appear to support the ion evaporation model (IEM) whereby small solvated ions are rejected from highly charged clusters. ${ }^{28,29}$ In some respects, that may be the only option available to highly charged ions of the type discussed here. Since the results show that a very significant fraction of the Coulomb barrier appears as centre-of-mass kinetic energy of the fragments, there may not be sufficient internal energy in highly charged ions to sustain extended sequences of evaporative steps that involve extensive intermolecular bond breaking. Therefore, ions take the lowest energy route which as the calculation show is the ejection of small ions.

\section{Conclusion}

By monitoring the appearance and disappearance of fragments from the decay of multiply charged ammonia clusters, $\left(\mathrm{NH}_{3}\right)_{n}{ }^{z+}$, where $z$ is $\leq 8$, it has been possible to identify decay pathways close to the Rayleigh instability limit where ions undergo charge separation due to Coulomb fission. As part of the assignment process, a series of calculations have also been undertaken, where firstly electrostatic theory appropriate for dielectric particles has been used to calculate the Coulomb barriers responsible for the release of kinetic energy following fragmentation. Secondly, these energy releases have been used to calculate the shapes of peak profiles expected of different fragmentation pathways, some of which can be eliminated due to a poor match with the experimental data. The experiments have provided a measure of the critical cluster size required for the onset of Coulomb instability at a given value of $z$, the total 
charge, and there is excellent agreement between the values recorded and results determined from the Rayleigh expression for the minimum number of molecules required to support $z$ charges.

\section{Acknowledgements}

$\mathrm{CH}$ and AJS would like to thank the University of Nottingham financial support and for the award of a research studentship to $\mathrm{CH}$, and AJS would like to thank the Leverhulme Trust for the award of an Emeritus Fellowship. The work also benefited from frequent discussions on the theory of electrostatic interactions with Prof. Elena Besley. 


\section{References}

(1) Rayleigh, Lord, On the equilibrium of liquid conducting masses charged with electricity. Philos. Mag. A 1882, 14, 184-186.

(2) Kreisle, D.; Leiter, K.; Echt, O.; Märk, T. D. Dissociation channels for multiply charged ammonia clusters. Z. Phys. D: At. Mol. Clusters 1986, 3, 319-322.

(3) Kreisle, D.; Echt, O.; Knapp, M.; Recknagel E.; Leiter, K.; Märk, T. D.; Saenz, J. J.;

Soler, J. M. Dissociation channels for multiply charged clusters, Phys. Rev. Lett. 1986, 56, $1551-1554$

(4) Echt, O.; Kreisle, D.; Recknagel, E.; Saenz, J. J.; Casero, R.; Soler, J. M. Dissociation channels of multiply charged van der Waals clusters. Phys. Rev. A 1988, 38, 3236-3248.

(5) Last, I.; Levy, Y.; Jortner, J. Fragmentation channels of large multiply charged clusters. $J$. Chem. Phys. 2005, 123, 154301.

(6) Mähr, I.; Zappa, F.; Denifl, S.; Kubala, D.; Echt, O.; Märk, T. D.; Scheier, P. Multiply charged neon clusters: Failure of the liquid drop model. Phys. Rev. Lett. 2007, 98, 023401.

(7) Hoener, M.; Bostedt, C.; Schorb, S.; Thomas, H.; Foucar, L.; Jagutzki, O.; SchmidtBöcking, H.; Dörner R.; Möller, T. From fission to explosion: Momentum-resolved survey over the Rayleigh instability barrier. Phys. Rev. A 2008, 78, 021201.

(8) Gotts, N. G.; Stace, A. J. Collision-induced Coulomb fragmentation in mass-selected $\left(\mathrm{CO}_{2}\right)_{n}{ }^{2+}$ clusters. Phys. Rev. Lett. 1991, 66, 21-24.

(9) Gotts, N. G.; Stace, A. J. Collision-induced Coulomb explosion in mass-selected triply charged benzene clusters. J. Chem. Phys. 1991, 95, 6175-6177.

(10) Gotts, N. G.; Lethbridge, P. G.; Stace, A. J. Observations of Coulomb explosion in doubly charged atomic and molecular clusters. J. Chem. Phys. 1992, 96, 408-421. 
(11) Wu, G.; Chen, X.; Stace, A. J.; Linse, P. Delayed asymmetric Coulomb fission of molecular clusters: Application of a dielectric liquid drop model. J. Chem. Phys. 2011 134, 031103.

(12) Chen, X.; Bichoutskaia, E.; Stace, A. J. Coulomb fission in dielectric dication clusters: Experiment and theory on steps that may underpin the electrospray mechanism. J. Phys. Chem. A 2013, 117, 3877-3886.

(13) Harris, C.; Baptiste J.; Lindgren, E. B.; Besley, E.; Stace, A. J. Coulomb fission in multiply charged molecular clusters: Experiment and theory. J. Chem. Phys. 2017, 146, 164302.

(14) Linse, P. Electrostatics in the presence of spherical dielectric discontinuities. J. Chem. Phys. 2008, 128, 214505.

(15) Bichoutskaia, E.; Boatwright, A. L.; Khachatourian, A.; Stace, A. J. Electrostatic analysis of the interactions between charged particles of dielectric materials. J. Chem. Phys. 2010, 133, 024105.

(16) Khachatourian, A.; Chan, H. K.; Stace, A. J.; Bichoutskaia,E. Electrostatic force between a charged sphere and a planar surface: A general solution for dielectric materials. J. Chem. Phys. 2014, 140, 074107.

(17) Kebarle, P.; Verkerk, U. H. Electrospray: From ions in solution to ions in the gas phase, what we know now. Mass Spectrom. Rev. 2009, 28, 898-917.

(18) Crotti, S.; Seraglia, R.; Traldi, P. Some thoughts on electrospray ionization mechanisms. Eur. J. Mass Spectrom. 2011, 17, 85-99.

(19) Grimm, R. L.; Beauchamp, J. L. Evaporation and discharge dynamics of highly charged droplets of heptane, octane, and p-xylene generated by electrospray ionization. Anal. Chem. 2002, 74, 6291-6297.

(20) Smith, J.N.; Flagan, R. C.; Beauchamp, J. L. Droplet evaporation and discharge dynamics in electrospray ionization. J. Phys. Chem. A, 2002, 106, 9957-9967. 
(21) Kebarle, P.; Peschke, M. On the mechanisms by which the charged ions produced by electrospray lead to gas phase ions. Anal. Chem. Acta, 2000, 406, 11-35.

(22) Hogan, Jr. C. J.; Biswas, P.; Chen, D. R. Charged droplet dynamics in the submicrometer size range. J. Phys. Chem. 2009, 113, 970-976.

(23) Loscertales, I. G.; de la Mora, F. Experiments on the kinetics of field evaporation of small ions from droplets. J. Chem. Phys. 1995, 103, 5041-5060.

(24) Gamero-Castano, M.; de la Mora, F. J. Kinetics of small ion evaporation from the charge and mass distribution of multiply charged clusters in electrospray. Mass Spectrom. 2000, 35, $790-803$

(25) Gamero-Castano, M.; de la Mora, F. Direct measurements of ion evaporation kinetics from electrified liquid surfaces. J. Chem. Phys. 2000, 113, 815-832.

(26) Dole, M.; Mack, L. L.; Hines, R. L. Molecular beams of macroions. J. Chem. Phys. 1968, $49,2240-2249$.

(27) Winger, B. A.; LightWahl, K. J.; Loo, R. R. O.; Udseth, H. R.; Smith, R. D. Observation and implications of high mass-to-charge ration ions from electrospray-ionization massspectrometry. J. Am. Soc. Mass Spectrom. 1993, 4, 536-545.

(28) Iribarne, J. V.; Thomson, B. A. On the evaporation of small ions from charged droplets. J. Chem. Phys. 1976, 64, 2287-2294.

(29) Thomson, B. A.; Iribarne, J. V. Field-induced ion evaporation from liquid surfaces at atmospheric-pressure J. Phys. Chem. 1979, 71, 44514463.

(30) Cooks, R. G.; Beynon, J. H; Caprioli, R. M.; G. R. Lester, G. R. Metastable Ions;(Elsevier: Amsterdam, 1973.

(31) Stace, A. J. Evidence of 2 stable forms of doubly and triply charged water cluster. Phys. Rev. Lett. 1988, 61, 306-309. 
(32) Stace, A. J. Possible ion-pairs in multiply charged water clusters. Chem. Phys. Lett. 1990, 174, 103-107.

(33) Coolbaugh, M. T.; Peifer, W. R.; Garvey, J. F. Ion-molecule chemistry within doubly charged ammonia clusters. Chem. Phys. Lett. 1989, 156, 19-23.

(34) Peifer, W. R.; Coolbaugh, M. T.; Garvey, J. F. Ion-molecule chemistry within triply charged ammonia clusters. J. Phys. Chem. 1989, 93, 4700-4703.

(35) Tolman, R. C. The superficial density of matter at a liquid-vapor boundary. J. Chem. Phys. 1949, $17,118-127$

(36) Tolman, R. C. The effect of droplet size on surface tension. J. Chem. Phys. 1949, 17, 333 337.

(37) Lu, H. M.; Jiang, Q. Size-dependent surface tension and Tolman's length of droplets. Langmuir 2005, 21, 779-781. 
Table 1

\begin{tabular}{|c|c|c|c|}
\hline Precursor cluster & Fragment pathway $\left(k^{+}\right)$ & $<K E R>/ \mathrm{eV}$ & $U_{\text {max }} / \mathrm{eV}$ \\
\hline$\left(\mathrm{NH}_{3}\right)_{52}{ }^{2+}$ & 11 & 0.99 & 0.97 \\
\hline & 12 & 0.99 & 0.98 \\
\hline & 13 & 0.99 & 0.98 \\
\hline & 14 & 0.99 & 0.98 \\
\hline
\end{tabular}

Table 2

\begin{tabular}{|c|c|c|}
\hline Charge state $z$ & Experimental $n_{\text {crit }}(z)$ & Calculated $n_{\text {crit }}(z)^{\#}$ \\
\hline $2+$ & 50 & 49 \\
\hline $3+$ & 116 & 111 \\
\hline $4+$ & 208 & 197 \\
\hline $5+$ & 302 & 308 \\
\hline $6+$ & 464 & 443 \\
\hline $7+$ & 630 & 603 \\
\hline $8+$ & 784 & 788 \\
\hline
\end{tabular}

\# calculated from the Rayleigh equation given in the text.

Table 3 


\begin{tabular}{|c|c|c|c|c|}
\hline Charge State & Critical range & Most probable & No. of & $U_{\max } / \mathrm{eV}$ \\
$z_{p}$ & & pathway & possible & \\
& & & fragments & \\
\hline $2+$ & 4 & $+1 /+1$ & 4 & $1.0^{\text {\# }}$ \\
\hline $3+$ & 8 & $+2 /+1$ & 3 & 1.6 \\
\hline $4+$ & 29 & $+3 /+1$ & 9 & 3.2 \\
\hline $5+$ & 105 & $+3 /+2$ & 9 & 4.0 \\
\hline $6+$ & 112 & $+4 /+2$ & 10 & 4.6 \\
\hline $7+$ & 72 & $+5 /+2$ & 21 & 3.4 \\
\hline $8+$ & & $+7 /+1$ & $\sim 3$ & \\
\hline
\end{tabular}

\# matches the experimental result

Table 4

\begin{tabular}{|c|c|c|}
\hline Precursor cluster & $\begin{array}{c}\text { Possible fragmentation } \\
\text { channels }\end{array}$ & $U_{\max } / \mathrm{eV}^{\#}$ \\
\hline$\left(\mathrm{NH}_{3}\right)_{492^{6+}}$ & $90^{2+}-99^{2+}$ & $\sim 4.0$ \\
\hline & & \\
\hline & $192^{3+}$ & $\sim 4.2$ \\
\hline
\end{tabular}


\# Barrier height calculated from electrostatic theory. Since the exact number varies slightly according to the precise size of a fragment, an average is given.

Table 5

\begin{tabular}{|c|c|c|}
\hline Precursor cluster & Possible fragments & $U_{\max } / \mathrm{eV}$ \\
\hline$\left(\mathrm{NH}_{3}\right)_{824^{8+}}$ & $31^{+}$ & 3.43 \\
\hline & $144^{2+}$ & 5.13 \\
\hline & $258^{3+}$ & 6.13 \\
\hline & $371^{4+}$ & 6.40 \\
\hline
\end{tabular}


Figure 1

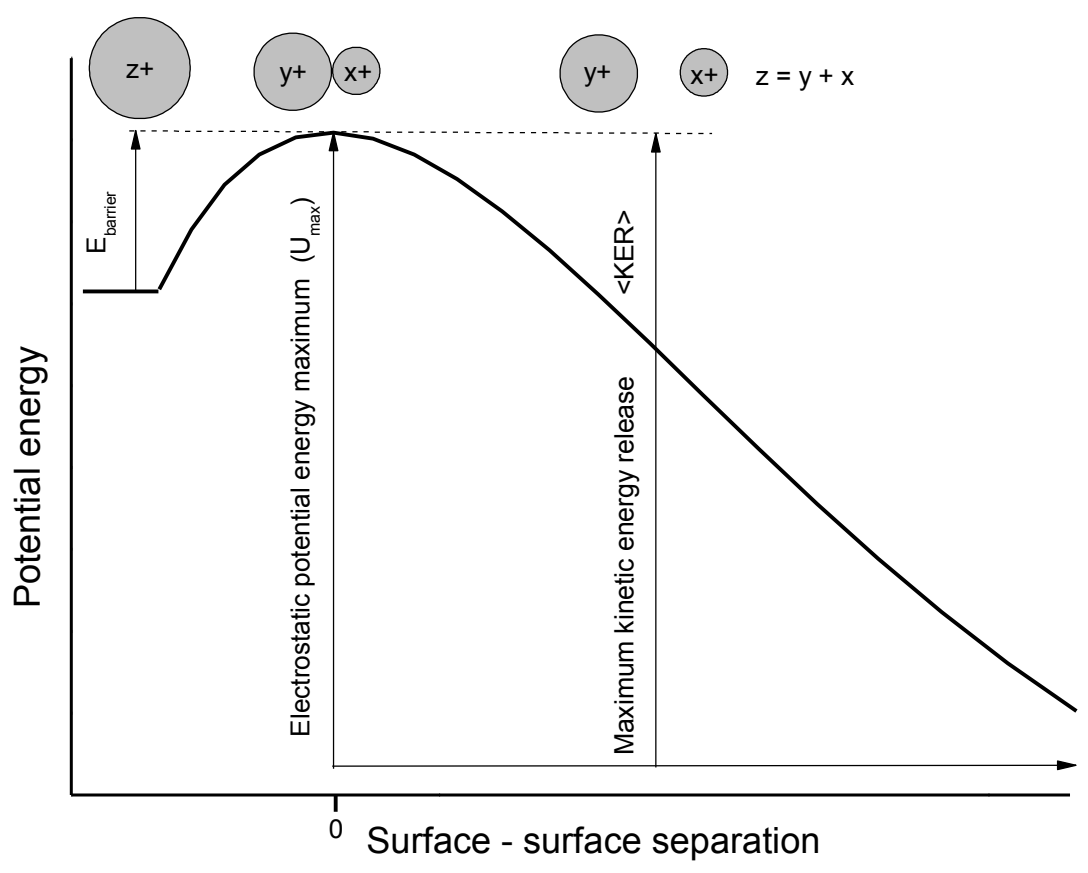

Figure 1. Potential energy curve for the coulomb fission of a multiply charged cluster carrying a charge of $z^{+}$fragmenting into two small clusters carrying charges of $y^{+}$and $x^{+}$. The maximum in the electrostatic potential energy curve corresponds to the point where the two charged fragments start to repel one another. $E_{\text {barrier }}$ denotes the energy barrier to fission. 
Figure 2

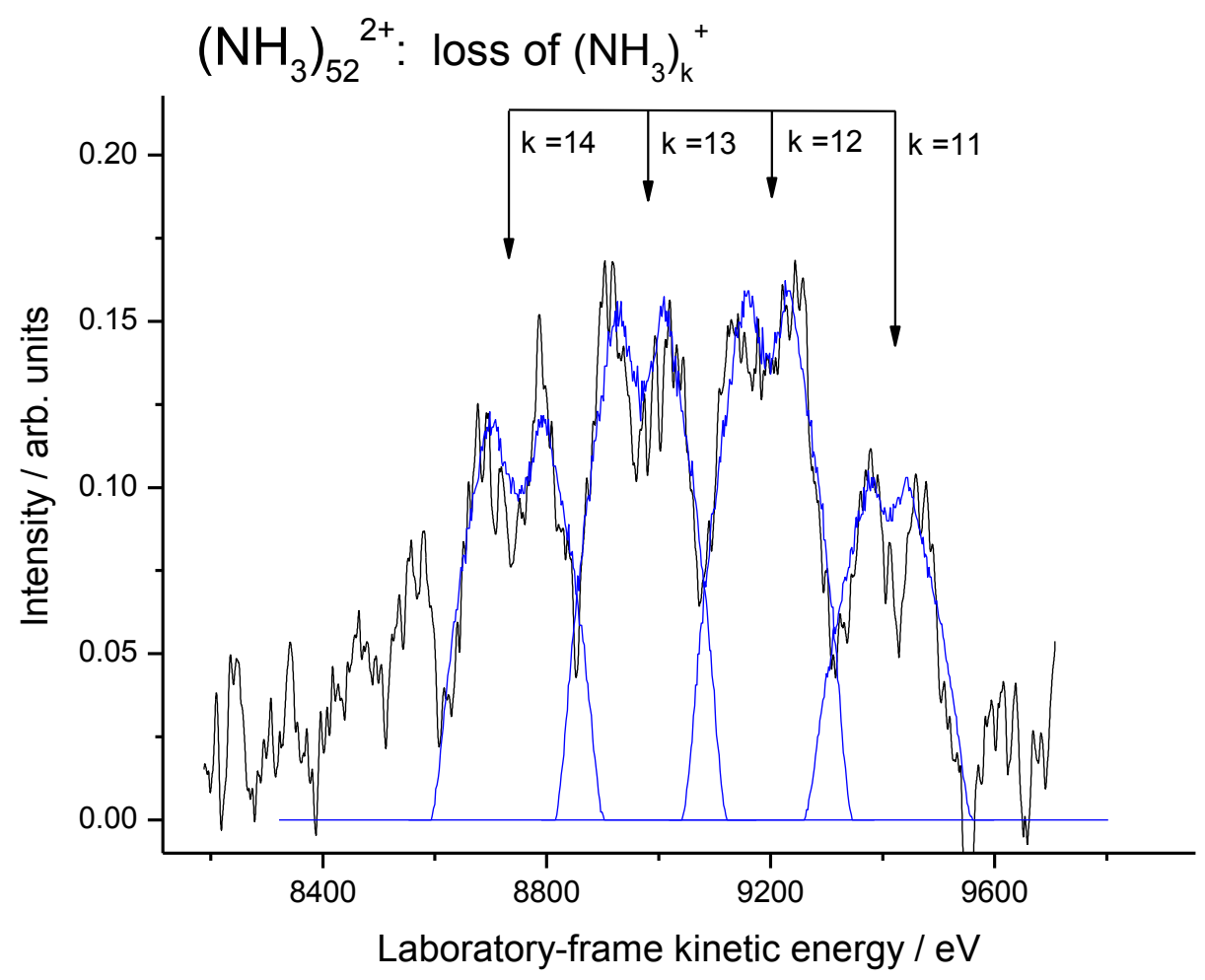


Figure 3

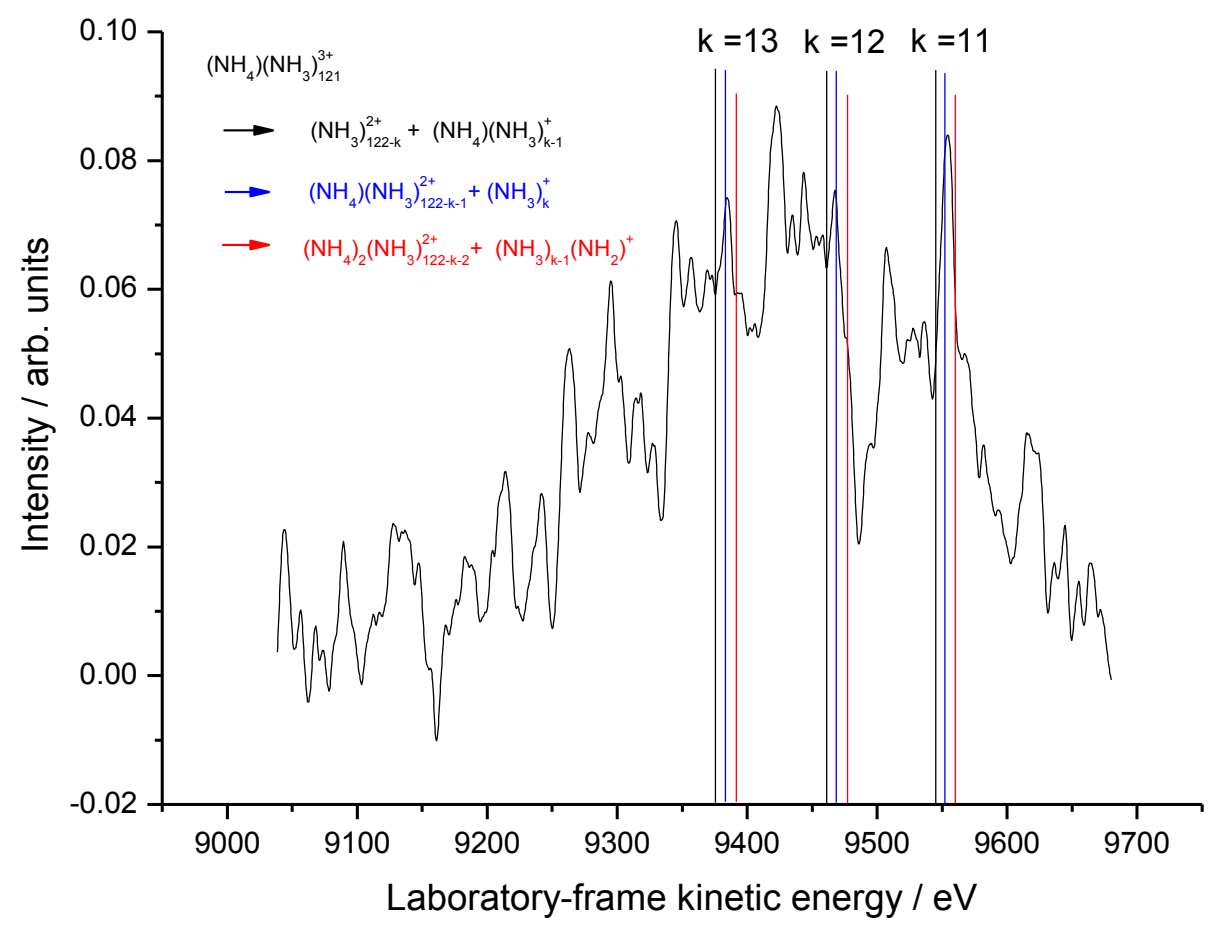


Figure 4

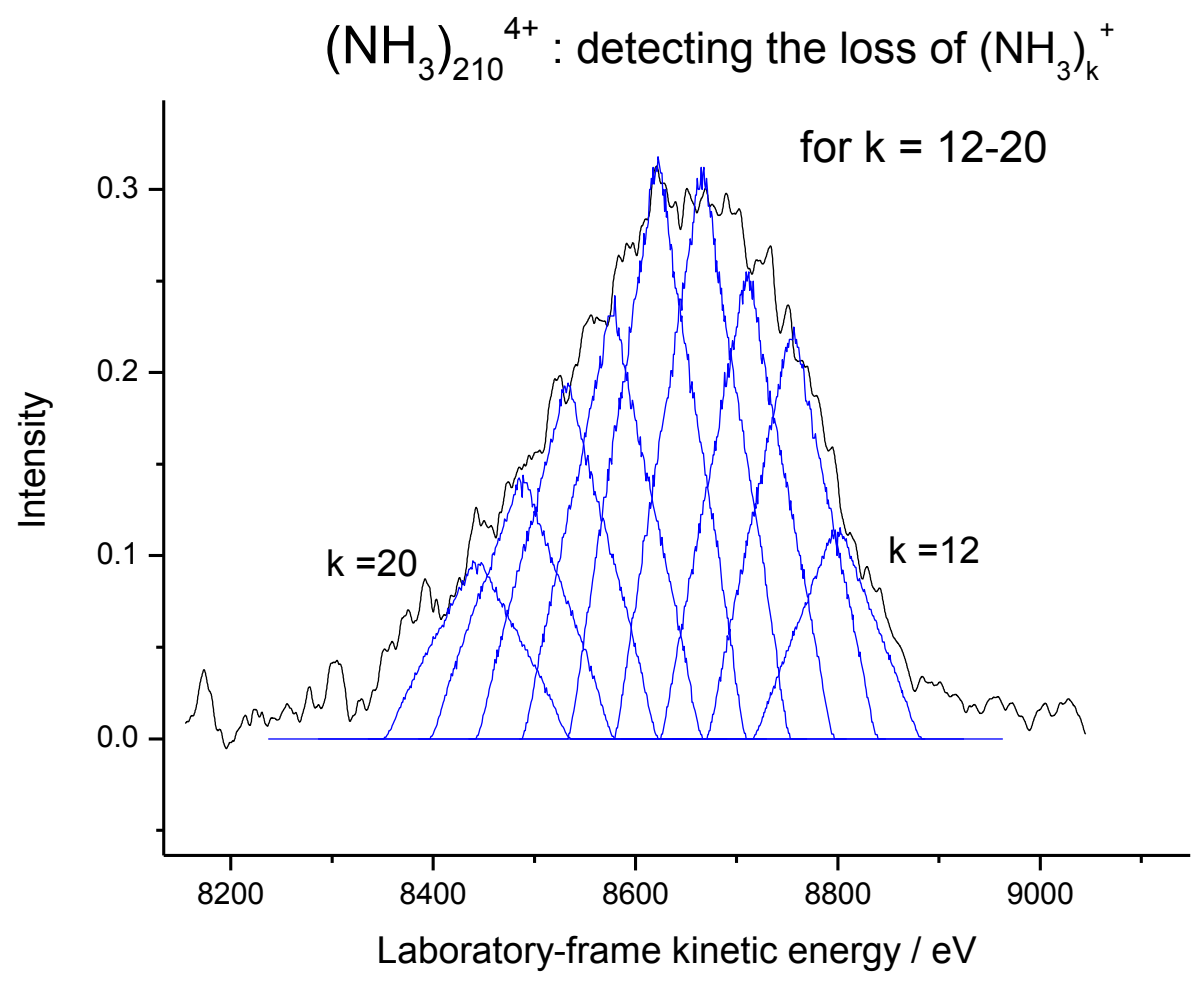


Figure 5

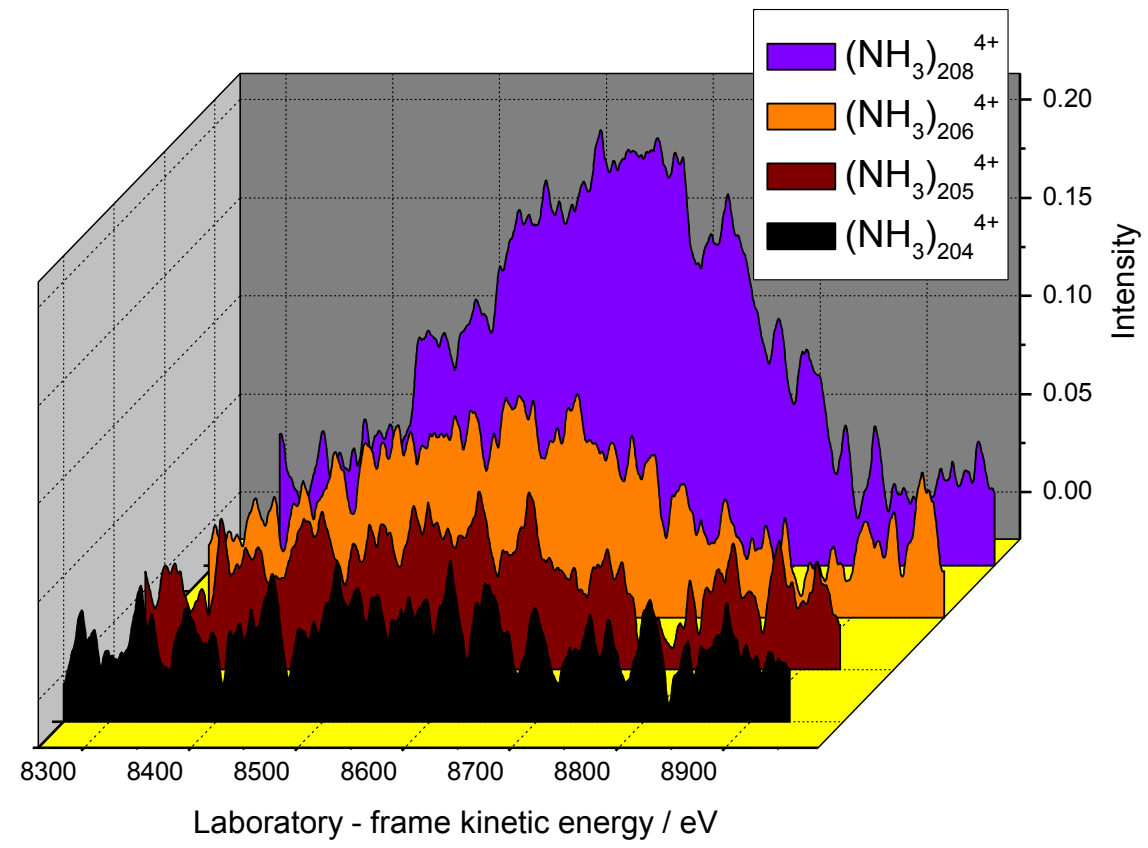


Figure 6

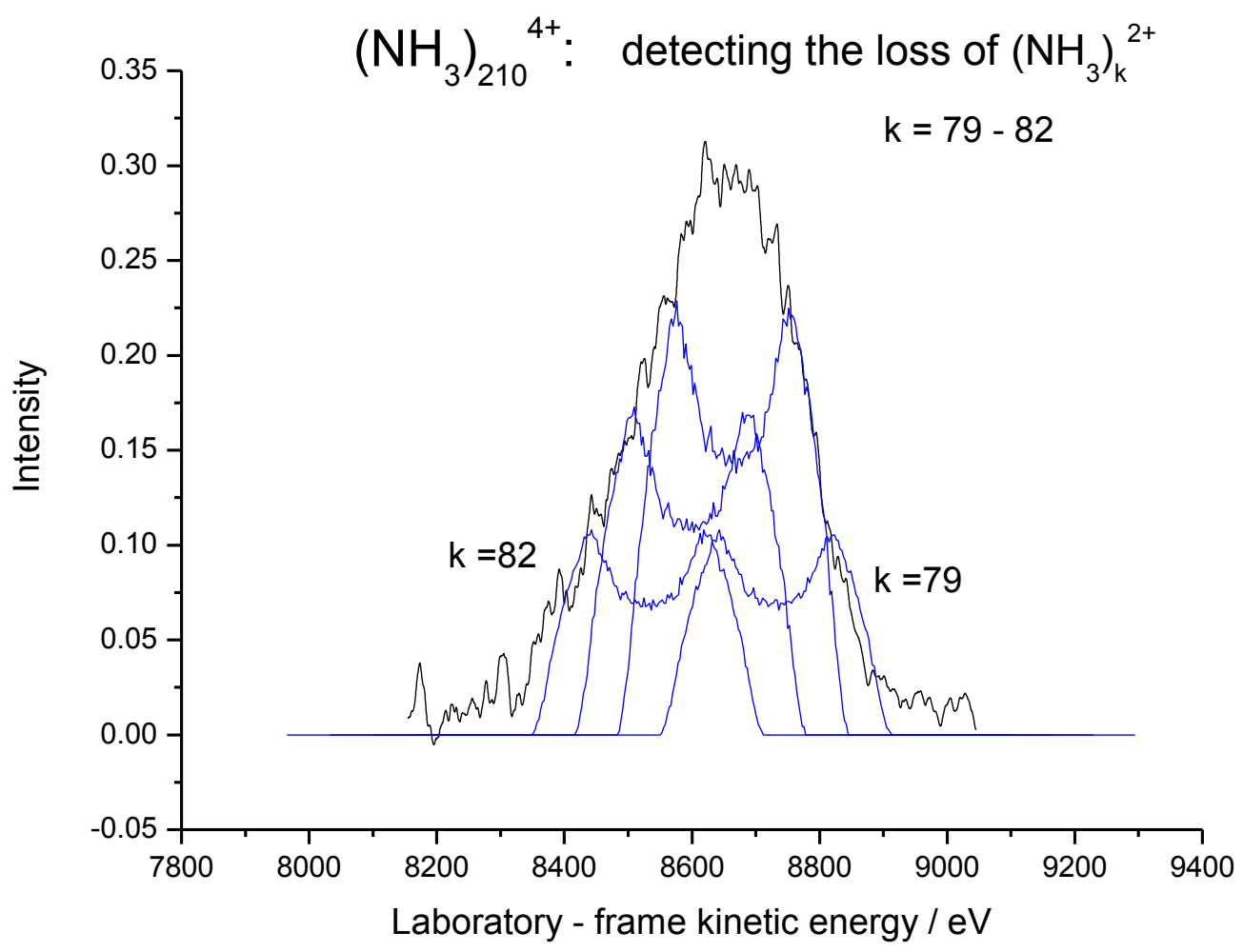


Figure 7

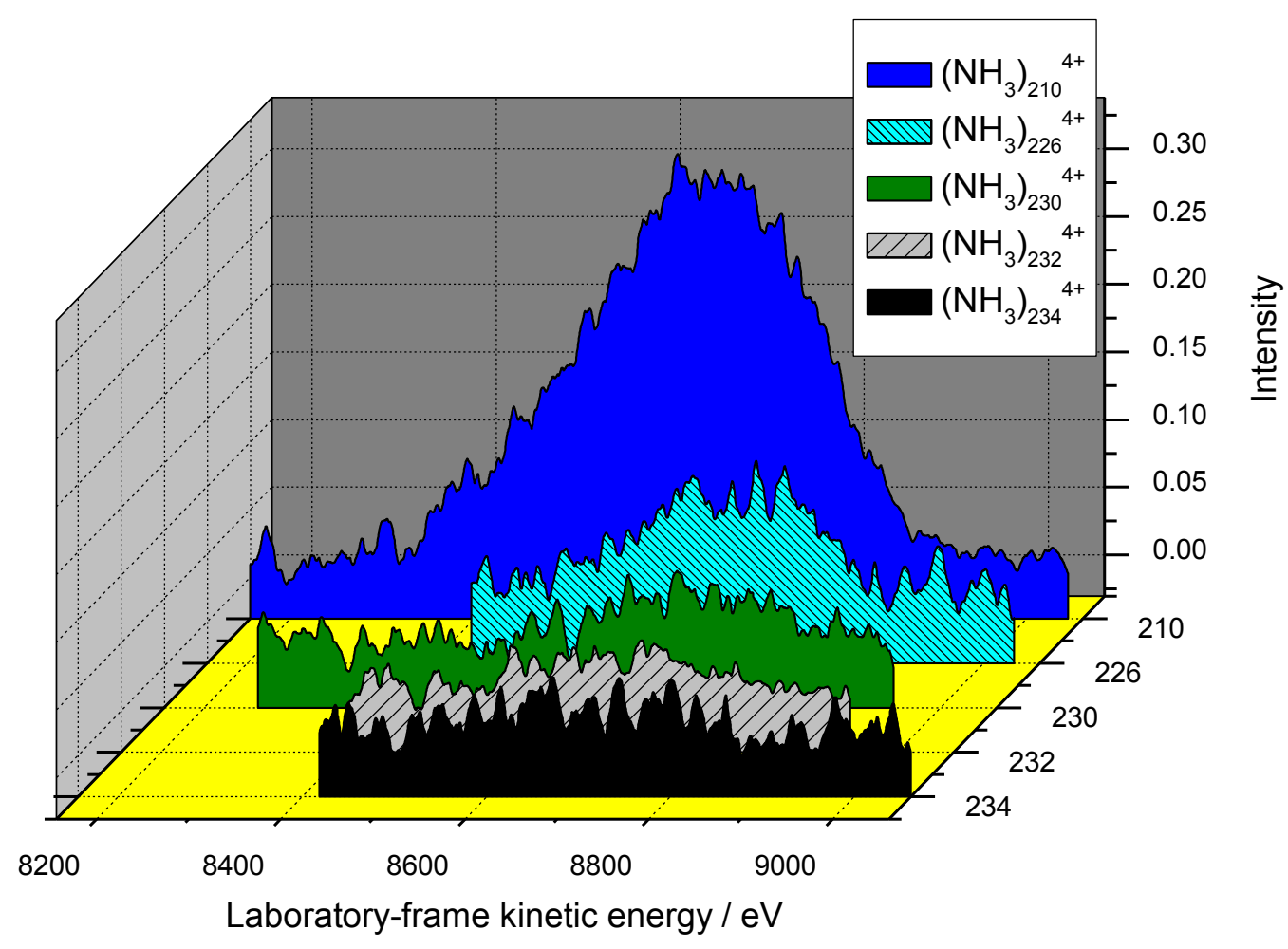


Figure 8

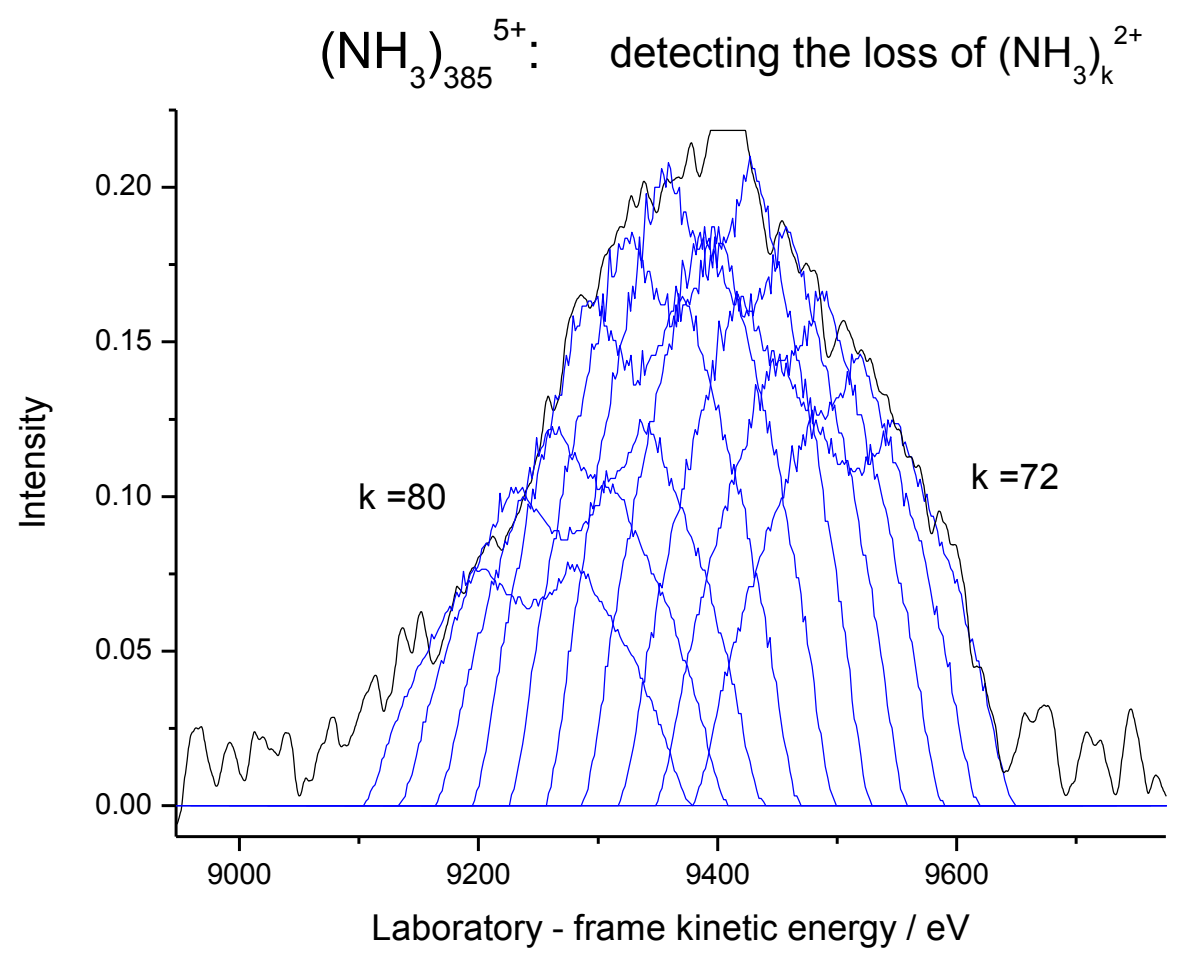


Figure 9

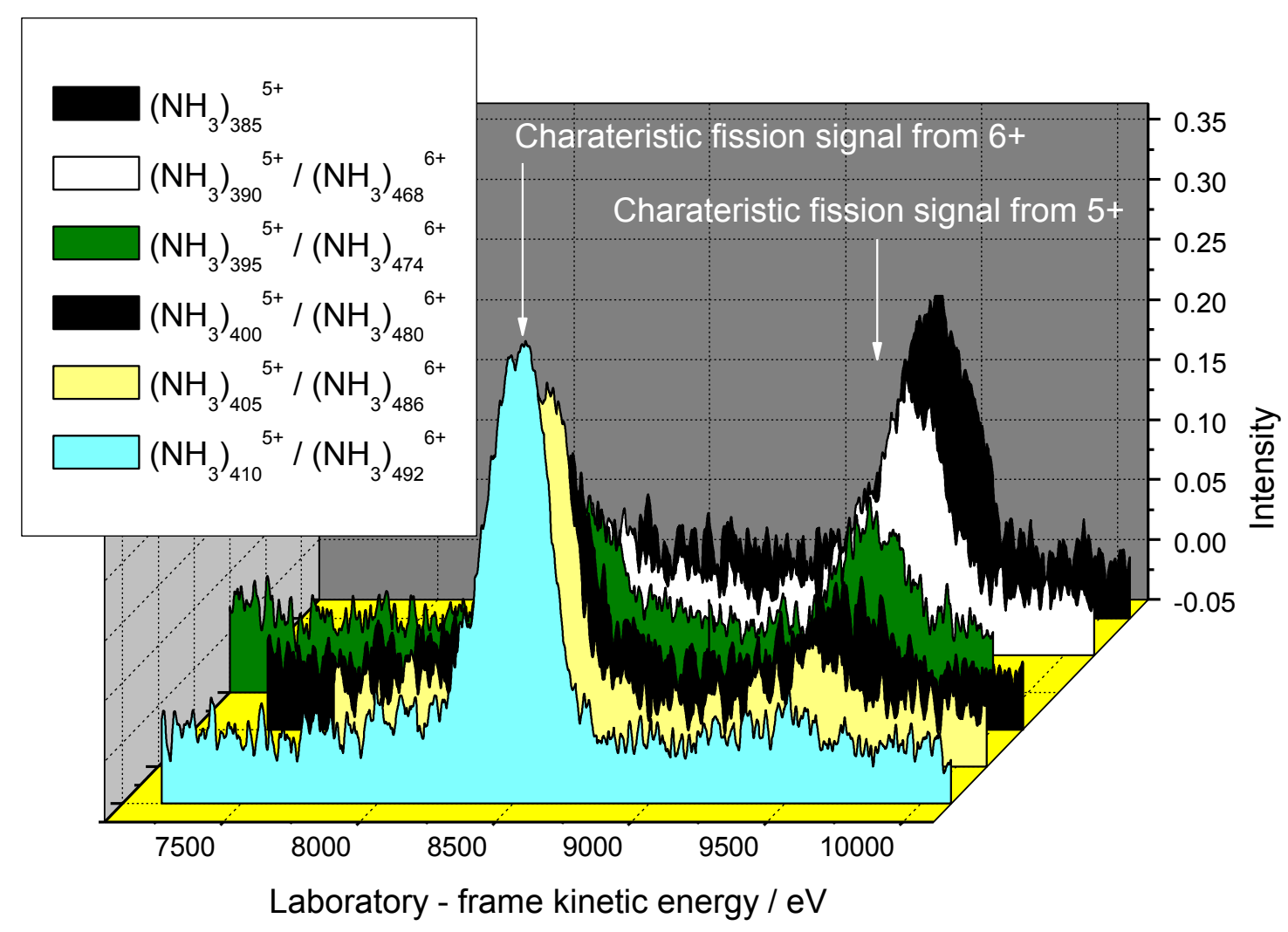


Figure 10

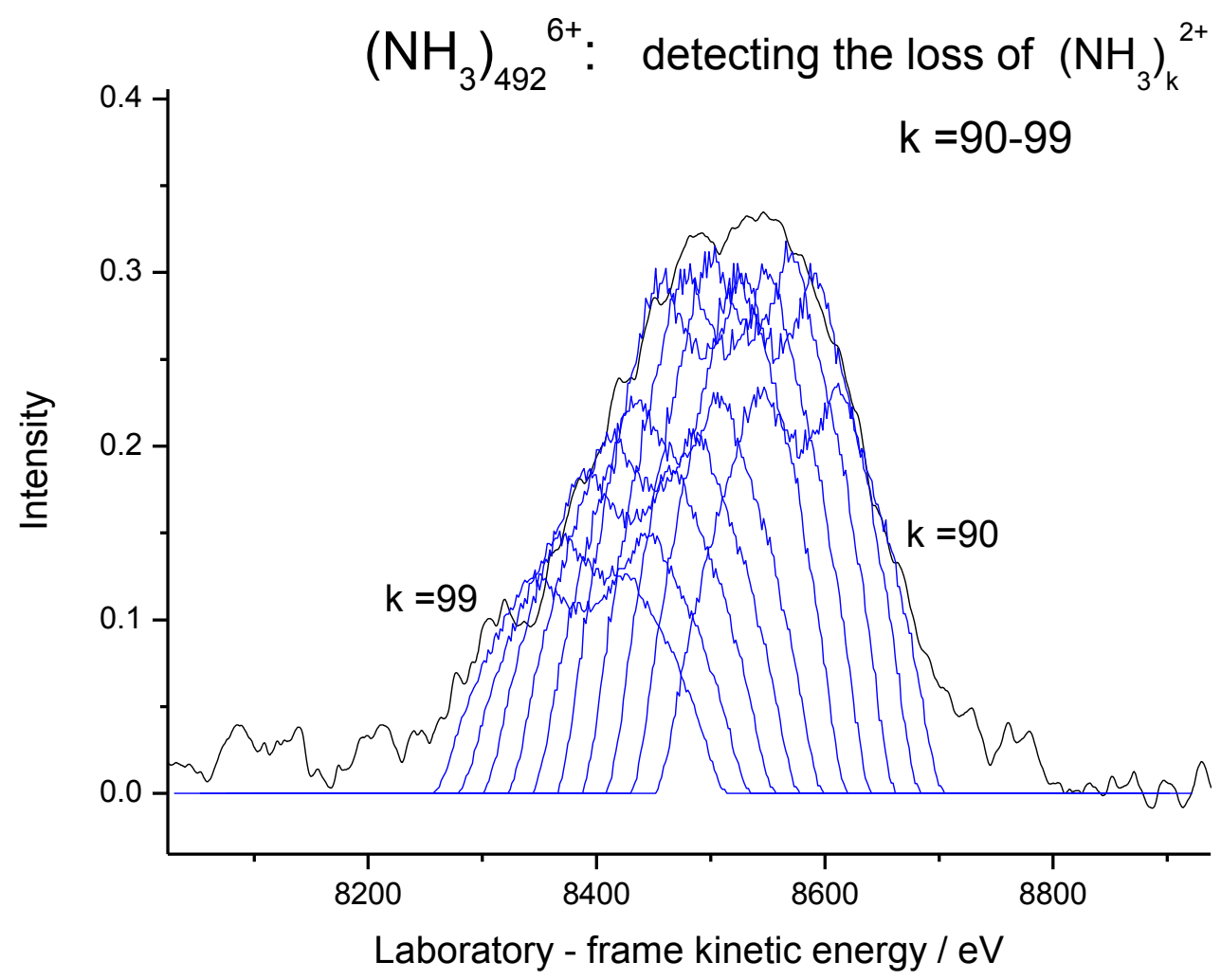


Figure 11

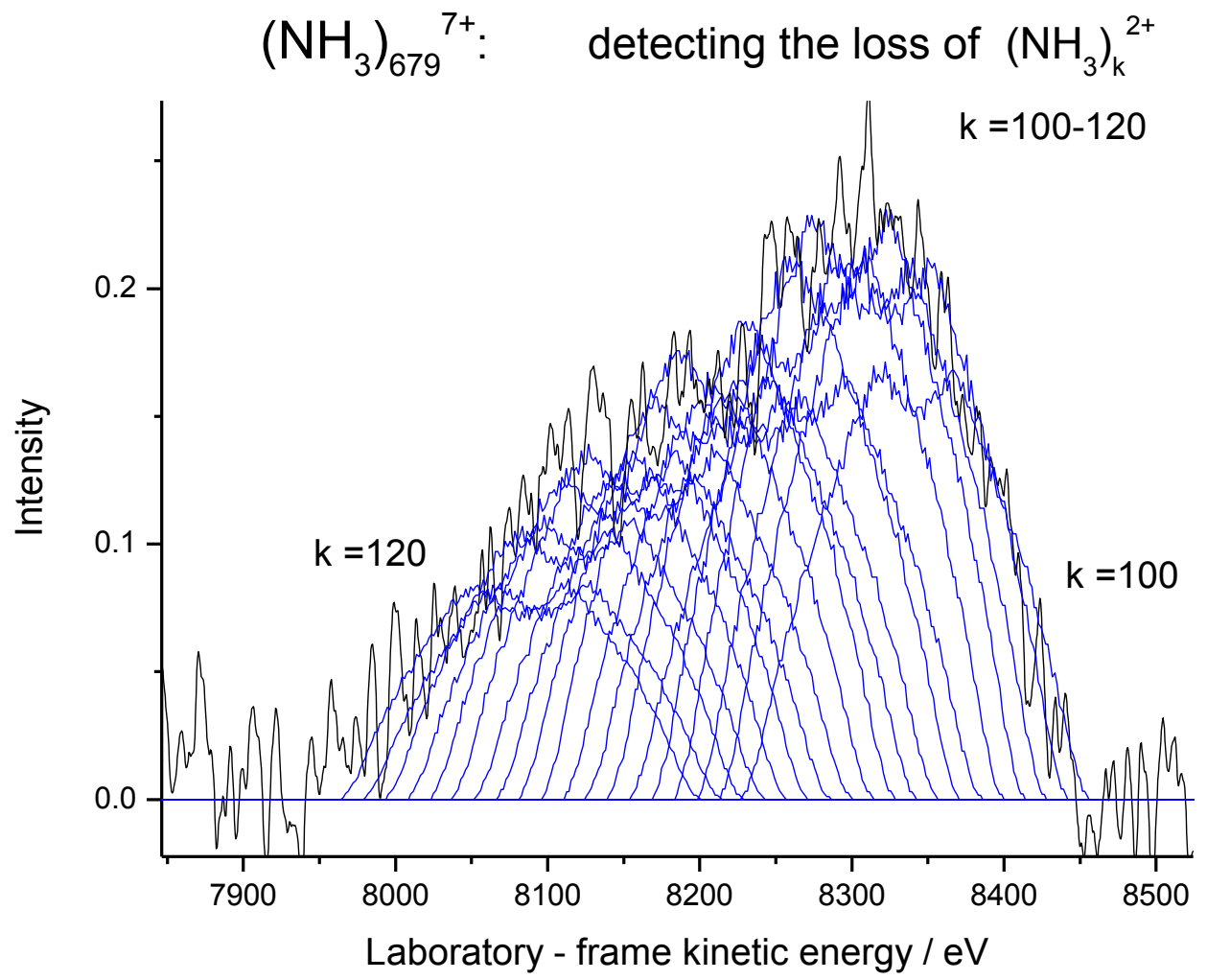


Figure 12

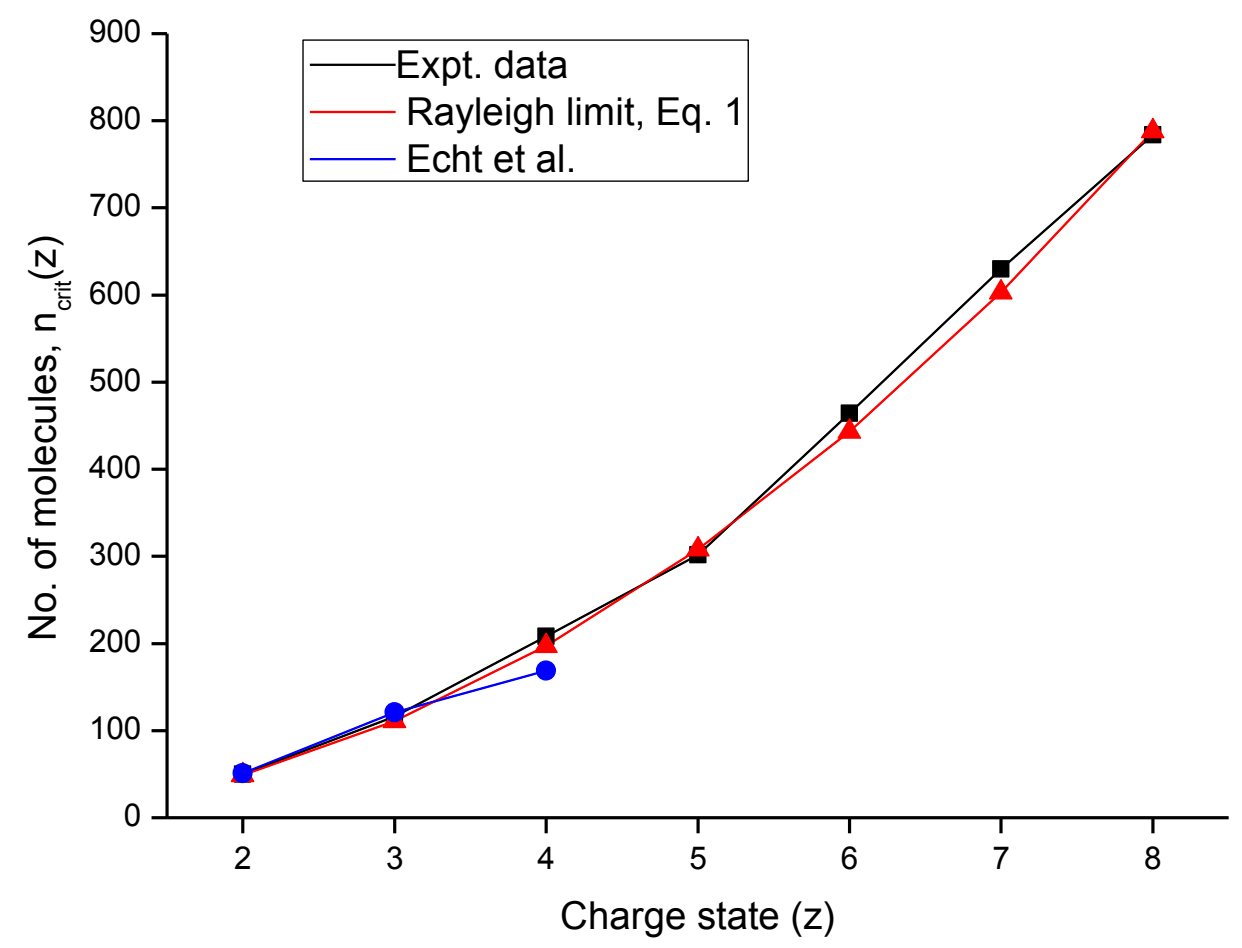


Figure 13

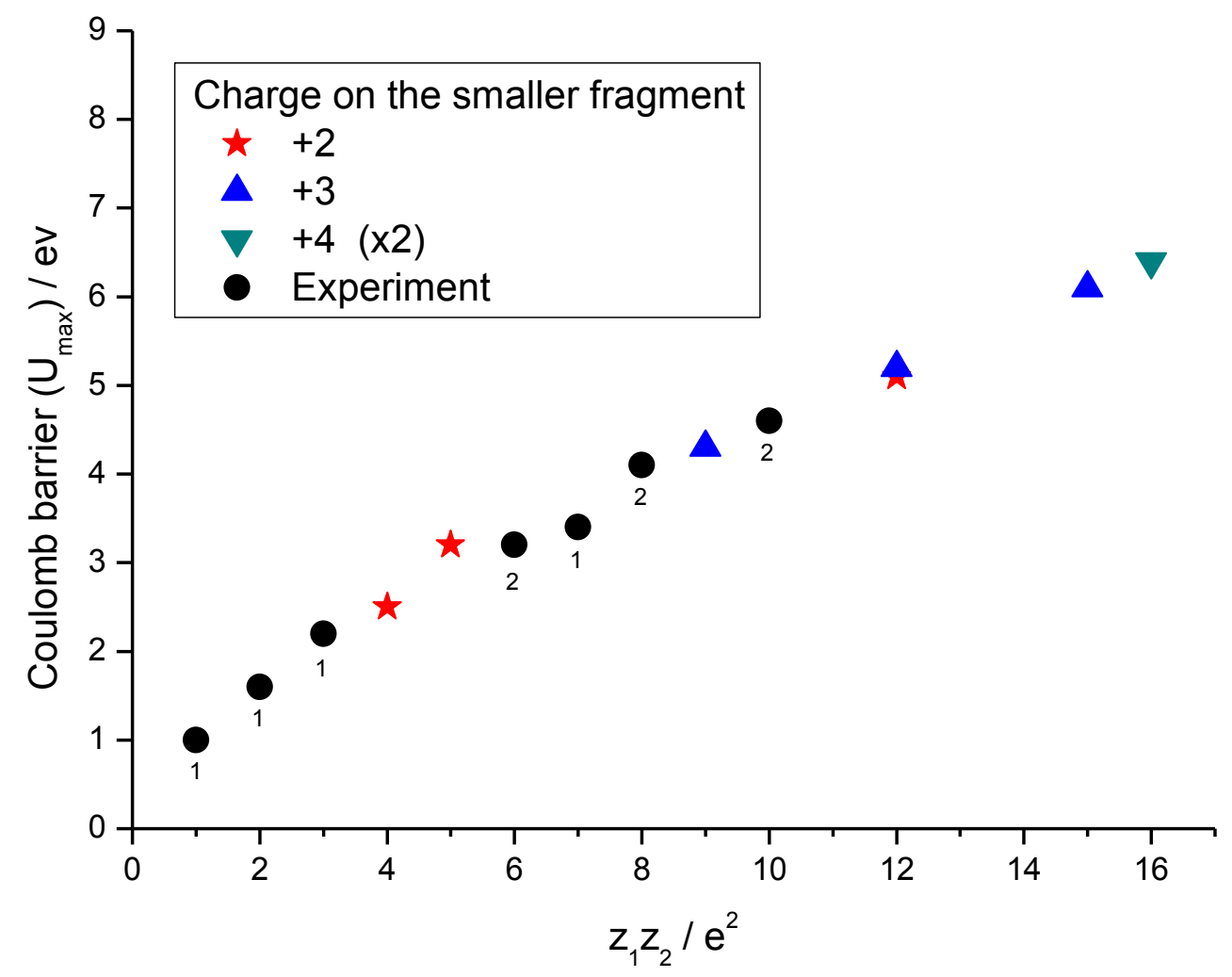

